\title{
Evaluación de un ensayo de RPC múltiple para diferenciar micobacterias del complejo Mycobacterium tuberculosis en un laboratorio de referencia
}

\author{
Nailet Arráiz R., Zolay Romay B. y Nelba Faría M.
}

\section{Evaluation of a multiplex PCR assay to differentiate mycobacteria of the Mycobacterium tuberculosis complex in a reference laboratory}

Mycobacteria that cause tuberculosis in animals and humans belong to the Mycobacterium tuberculosis complex. Techniques for conventional diagnosis are time-consuming and do not differentiate between different strains belonging to the M. tuberculosis complex. The aim of this study was to evaluate a multiplex PCR assay applicable to mycobacteria in culture with the capacity to differentiate different strains belonging to the $M$. tuberculosis complex in a reference laboratory. Primers based on genomics regions of difference (RD) consisting in DNA segments that are present in M. tuberculosis, but differentially deleted in several members of $M$. tuberculosis complex were used in a PCR assay. The test was applied to 86 clinical isolates of mycobacteria. The pattern of amplification allowed differentiating between $M$. tuberculosis, M. bovis and $M$. bovis BCG in a single PCR reaction. This PCR multiplex assay may be used in a Reference Laboratory of Tuberculosis Diagnosis as a complementary test to differentiate mycobacteria strains belonging to the $M$. tuberculosis complex. This test significantly reduces the time period between culture and strain identification, and thus for could favor the adoption of better strain specific antimycobacterial regimens as well as identification of zoonotic transmission of $M$. bovis to humans.

Key words: Mycobacterium tuberculosis complex, multiplex PCR, Mycobacterium bovis, RD1, RD4, reference laboratory.

Palabras claves: Complejo Mycobacterium tuberculosis, RPC múltiple, Mycobacterium bovis, RD1, RD4, laboratorio de referencia.

\section{Introducción}

$\mathrm{L}$ as enfermedades causadas por micobacterias del complejo Mycobacterium tuberculosis, siguen constituyendo una fuente importante de morbilidad y mortalidad en el mundo. El complejo $M$. tuberculosis incluye micobacterias de crecimiento lento causantes de tuberculosis en animales y humanos como: Mycobacterium tuberculosis, Mycobacterium bovis, Mycobacterium bovis variedad BCG, Mycobacterium africanum, Mycobacterium microti y Mycobacterium canetti, una subespecie de M. tuberculosis reportada en tuberculosis humana ${ }^{1-3}$.

Cerca del $90 \%$ de las personas afectadas por $M$. tuberculosis se concentran en los países tropicales en vías de desarrollo ${ }^{4}$. En Venezuela, la tuberculosis se ubica entre las primeras veinte causas de muerte, con una incidencia estimada entre 25 y 49 casos/100.000 habitantes, siendo los estados más afectados el Distrito Capital, Zulia, Miranda y Delta Amacuro5.

Aunque $M$. tuberculosis se conoce como el agente causal de tuberculosis humana, se ha reconocido la importancia epidemiológica de la transmisión zoonótica de $M$. bovis a humanos debido, potencialmente, al contacto directo de humanos con animales infectados, o al consumo de productos provenientes de estos animales $^{6-8}$. También se ha reportado coinfección con M. bovis variedad BCG y M. bovis en pacientes infectados con virus de inmunodeficiencia humana (VIH) ${ }^{9,10}$.

El diagnóstico de tuberculosis descansa en la baciloscopia, el cultivo y subcultivo, seguido de una batería de pruebas bioquímicas para la identificación de la especie causante de la infección; sin embargo, el reporte de resultados es muy tardío y no siempre se logra distinguir entre los miembros del complejo $M$. tuberculosis, lo cual puede confundir el diagnóstico y
Universidad del Zulia, Maracaibo, Venezuela Facultad de Medicina Centro de Investigaciones Endocrino-Metabólicas "Dr. Félix Gómez" Sección de Biología Molecular y Escuela de Bioanálisis (NAR)

Servicio Autónomo del Hospital de Maracaibo Estado de Zulia, Maracaibo, Venezuela Coordinación del Programa Regional de Control de Tuberculosis

Recibido: 11 mayo 2006 Aceptado: 3 enero 2007

Correspondencia a: Nailet Arráiz Rodríguez narraiz@cantv.net 
la epidemiología ${ }^{11}$. En los últimos años se han utilizado algunas técnicas moleculares disponibles comercialmente, basadas en amplificación por reacción de polimerasa en cadena (RPC) directamente sobre muestras clínicas ${ }^{12-14}$ o a partir de cultivos ${ }^{14}$; sin embargo, estos ensayos están generalmente dirigidos a la identificación de una especie dada y no permiten distinguir entre miembros del complejo $M$. tuberculosis.

El alto grado de conservación de secuencias de ADN entre las micobacterias del complejo M. tuberculosis, ha limitado el desarrollo de herramientas de diagnóstico molecular para identificar miembros individuales del complejo $M$. tuberculosis; no obstante, estudios filogenéticos recientes han puesto en evidencia regiones del genoma que exhiben diversos re-arreglos o múltiples polimorfismos, que han sido colectivamente designados como regiones de diferencia (RD o "regions of difference", por sus siglas en inglés) ${ }^{15-19}$. La comparación del ADN total de la cepa de referencia de $M$. tuberculosis H37RV con el genoma de otras micobacterias del complejo, ha revelado la existencia de $16 \mathrm{RD}$, de un tamaño promedio entre 2 y $12,7 \mathrm{~Kb}$, presentes en el genoma de $M$. tuberculosis, pero ausentes en $M$. bovis, M. bovis variedad $\mathrm{BCG}^{19}$, y en otros miembros del complejo ${ }^{15,16,19,20}$.

Debido a que los loci RD parecen haberse acumulado secuencialmente y están diferencialmente distribuidos entre varios grupos de micobacterias del complejo M. tuberculosis, éstos han sido propuestos como marcadores genéticos para diferenciar entre subespecies de este complejo ${ }^{15,20-23}$.

El objetivo del presente trabajo fue evaluar un protocolo de RPC múltiple, incorporando secuencias de oligonucleótidos partidores dirigidos a los loci RD1 y y RD4, ambos presentes en el genoma de $M$. tuberculosis, pero diferencialmente eliminados en M. bovis y
M. bovis variedad BCG, con la finalidad de contribuir a la identificación diferencial de estas micobacterias, a partir de cultivos obtenidos en un laboratorio de referencia para el diagnóstico de tuberculosis.

\section{Material y Método}

Se evaluaron 86 aislados clínicos: 60 cepas del complejo M. tuberculosis: M. tuberculosis (40), M. bovis (14) y M. bovis variedad BCG (6), y 26 cepas del complejo no tuberculosis: M. avium (5), M. flavescens (4), M. gordonae (4), M. kansasii (4), M. szulgai (3), M. scrofulaceum (3), M. chelonae (2) y M. terrae (1). Los aislados clínicos corresponden a cultivos de muestras de esputo de pacientes con clínica de tuberculosis, provenientes de Servicios de Neumonología y Tisiología de Centros de Salud del Estado Zulia, Venezuela, cuyas muestras fueron referidas al laboratorio de Referencia de Diagnóstico de Tuberculosis, con sede en el Servicio Autónomo del Hospital Universitario de Maracaibo (SAHUM), el cual está adscrito al Programa Regional de Control de Tuberculosis.

Oligonucleótidos utilizados en este estudio. En la Tabla 1 se muestra la secuencia de oligonucleótidos partidores empleados y se especifica el tamaño de los productos de RPC esperados. Para la diferenciación de micobacterias del complejo M. tuberculosis y no tuberculosis, se utilizó un ensayo de RPC doble previamente estandarizado en el laboratorio ${ }^{24}$, basados en secuencias cuya especificidad individual fue previamente reportada ${ }^{23}$. El ensayo incluyó un par de oligonucleótidos (16S ARNrAF y 16S ARNrAR) dirigidos a secuencias conservadas del gen $16 \mathrm{~S}$ ARNr que permiten amplificar un fragmento de 543 pares de bases (pb) en cualquier especie micobacteriana, y un segundo par

Tabla 1. Oligonucleótidos utilizados en este estudio

\begin{tabular}{|c|c|c|c|}
\hline Nombre del oligo NT & Secuencia de nucleótidos & Descripción & $\begin{array}{l}\text { Tamaño del } \\
\text { oducto de RPC }\end{array}$ \\
\hline Rv16SrDNAF & 5' ACGGTGGGTACTAGGTGTGGGTTTC $3^{\prime}$ & Gen de ADNr 16S. Género específico & $543 p b$ \\
\hline Rv16SrDNAR & 5' TCTGCGATTACTAGCGACTCCGACTTCA $3^{\prime}$ & & \\
\hline Rv0577F & 5' ATGCCCAAGAGAAGCGAATACAGGCAA $3^{\prime}$ & Específico del complejo M. tuberculosis & $786 \mathrm{pb}$ \\
\hline Rv0577R & 5' CTATTGCTGCGGTGCGGGCTTCAA $3^{\prime}$ & & \\
\hline Rv1510F & 5' GTGCGCTCCACCCAAATAGTTGC 3' & Región de diferencia RD4. Ausente en $M$. bovis y $M$. bovis $B C G$ & $1033 \mathrm{pb}$ \\
\hline Rv1510R & $5^{\prime}$ TGTCGACCTGGGGCACAAATCAGT C $3^{\prime}$ & & \\
\hline Rv3877/8F & 5' CGACGGGTCTGACGGCCAAACTCATC $3^{\prime}$ & Región de diferencia RD1. Ausente en $M$. bovis BCG & $999 \mathrm{pb}$ \\
\hline Rv3877/8R & 5' CTTGCTCGGTGGCCGGTTTTTCAGC 3' & & \\
\hline
\end{tabular}


de oligonucleótidos (Rv0577F y Rv0577R) dirigidos a la secuencia de la región codificante del gen Rv0577 que amplifican un segmento de 786 pb, sólo en micobacterias del complejo M. tuberculosis ${ }^{23,24}$.

Una vez detectadas las cepas del complejo $M$. tuberculosis, se aplicó un segundo ensayo de RPC múltiple, que también incluyó los oligonucleótidos del gen Rv0577, y otros dirigidos a los loci RD1 y RD4 ${ }^{15,22,23}$. Los oligonucleótidos partidores dirigidos al gen Rv1510 del locus RD4, se utilizaron para diferenciar M. tuberculosis de $M$. bovis y M. bovis variedad BCG, esperando un fragmento de 1033 pb sólo en $M$. tuberculosis, pero no en $M$. bovis, ni $M$. bovis variedad BCG. La ausencia de fragmento de 1033 pb revela la presencia de $M$. bovis (incluyendo M. bovis variedad BCG), de manera que para diferenciar entre $M$. bovis y $M$. bovis variedad BCG, se incluyeron los oligonucleótidos Rv3877/78F y Rv3877/78R dirigidos al locus RD1 localizado entre los genes Rv3877 y Rv3878, el cual está ausente en $M$. bovis variedad BCG, por lo cual el fragmento de 999 pb será amplificado del genoma de $M$. bovis y $M$. tuberculosis, pero no de $M$. bovis variedad BCG. Los oligonucleótidos fueron sintetizados por Maxim Biotech, INC, EUA.

Extracción y purificación de ADN genómico de colonias de micobacterias. Se siguió un procedimiento de lisis enzimática ${ }^{25}$ con modificaciones. Se tomó una asada de una colonia micobacteriana, se colocó en tubo conteniendo $500 \mu \mathrm{l}$ de tampón TE (10 mM Tris$\mathrm{HCl}, \mathrm{pH}$ 8, 1 mM EDTA, pH 8). Se inactivó con calor a $90{ }^{\circ} \mathrm{C}$ durante 1 hora. Se agregó $100 \mu \mathrm{l}$ de lisozima $10 \mathrm{mg} / \mu \mathrm{l}$ y se incubó a $37^{\circ} \mathrm{C}$ por una hora. Se agregó 30 $\mu \mathrm{l}$ de SDS $10 \%$ y $10 \mu \mathrm{l}$ de proteinasa K $10 \mathrm{mg} / \mu \mathrm{l}$, se incubó a $65^{\circ} \mathrm{C}$ durante dos horas. Se hizo una extracción con $500 \mu \mathrm{l}$ de cloroformo, la fase acuosa se transfirió a otro tubo y se precipitó con dos volúmenes de etanol $100 \%$. El sedimento se lavó con etanol $70 \%$ y se resuspendió en $30 \mu 1$ de tampón TE. Se utilizó 2,0 $\mu 1$ de la muestra para ensayos de amplificación.

Condiciones de amplificación por RPC. Para el ensayo inicial de RPC doble se utilizaron condiciones de amplificación descritas previamente ${ }^{24}$. Se estandarizaron las condiciones de amplificación simultánea con los oligonucleótidos dirigidos a secuencias Rv0577, Rv1510 y Rv3877/8 incorporados en ensayo de RPC múltiple y se seleccionó $57{ }^{\circ} \mathrm{C}$ como temperatura de alineamiento óptima en el programa de amplificación. Se preparó la mezcla de reacción para un volumen final de $50 \mu \mathrm{l}$, consistiendo en $5 \mu \mathrm{l}$ de tampón 10X Taq ADN polimerasa (PROMEGA), 1,5 $\mathrm{mM} \mathrm{MgCl}_{2}, 200 \mathrm{mM}$ cada desoxirribonucleótido (dATP, dCTP, dGTP y dTTP) y 20 pmoles cada oligonucleótido. Se utilizó 0,25 U de Taq ADN polimerasa (PROMEGA) para cada reacción. El programa de amplificación consistió en tres minutos de denaturación inicial a $94{ }^{\circ} \mathrm{C}$ y 30 ciclos de amplificación de 45 segundos a $94{ }^{\circ} \mathrm{C}, 45$ segundos a $57^{\circ} \mathrm{C}$, 45 segundos a $72{ }^{\circ} \mathrm{C}$ y un paso final de amplificación a $72{ }^{\circ} \mathrm{C}$ durante diez minutos. Las reacciones se llevaron a cabo en un termociclador MJ Research PTC-100Ô. Como control negativo y de riesgos de contaminación cruzada durante los procedimientos de extracción y amplificación, se procesaron en paralelo colonias de Escherichia coli.

Los productos de RPC ( $5 \mu \mathrm{l})$ se analizaron en geles de agarosa $1 \%$. Los geles fueron teñidos con bromuro de etidio, visualizados en transiluminador ultravioleta y fotografiados con sistema de fotodocumentación DigiDoc UVP. Se utilizó marcador de peso molecular PCR markers de PROMEGA (1.000, 750, 500, 300, 150 y $50 \mathrm{pb})$.

\section{Resultados}

El protocolo de identificación de micobacterias basado en dos ensayos de amplificación por RPC (doble y múltiple), fue aplicado sobre aislados clínicos procesados al azar, consistiendo en cepas del complejo $M$. tuberculosis (M. tuberculosis, M. bovis y M. bovis variedad BCG) y no tuberculosis ( $M$. avium, $M$. flavescens, M. gordonae, M. kansasii, M. szulgai, M. scrofulaceum, M. chelonae y $M$. terrae), previamente identificadas en un laboratorio de referencia de diagnóstico de tuberculosis. Como se observa en la Figura 1, al aplicar el ensayo de RPC doble, el fragmento de $543 \mathrm{pb}$ correspondiente a la región del gen de ARN ribosomal común a todas las micobacterias, fue detectado en el genoma de las 86 cepas analizadas, incluyendo las del complejo M. tuberculosis y no tuberculosis, mientras que el fragmento de 786 pb correspondiente al marco de lectura Rv0577 permitió identificar aislados clínicos del complejo M. tuberculosis [M. tuberculosis $(\mathrm{n}=40)$, M. bovis $(\mathrm{n}=14)$ y M. bovis variedad BCG $(\mathrm{n}=6)$, confirmando que este gen está restringido al complejo $M$. tuberculosis ${ }^{17,23}$ y reiterando la utilidad de este ensayo para diferenciar entre cepas del complejo M. tuberculosis y no tuberculo$\operatorname{sis}^{23,24}$.

Cuando las muestras que resultaron positivas para el complejo $M$. tuberculosis fueron analizadas con el segundo ensayo (RPC múltiple), se observó un producto de RPC de $1.033 \mathrm{pb}$ correspondiente a una región del gen Rv1510 (locus RD4) sólo en M. tuberculosis, pero no en $M$. bovis ni en M. bovis variedad BCG (Figura 2), consistente con la eliminación de esta región en el genoma de estas especies micobacterianas ${ }^{15,19,23}$, de manera que la ausencia del fragmento de $1.033 \mathrm{pb}$ permite diferenciar M. bovis (incluyendo $M$. 
Figura 1. Ensayo de RPC doble para detectar micobacterias del complejo M. tuberculosis. Se observan productos de RPC de 543 pb del gen $16 \mathrm{~S}$ ADNr en todas las micobacterias y fragmentos de 786 pb del gen Rv0577 sólo en aislados clínicos del complejo M. tuberculosis. Líneas: 3, 4, 9, 15: M. tuberculosis; 7, 17,18: M. bovis; 10: $M$. bovis BCG; 1: $M$. smegmatis; 2: $M$. avium; $5: M$. flavescens; 8: M. gordonae; 11: M. kansasii; 13: M. szulgai; 14: M. scrofulaceum; 16: M. chelonae; 6 y 12: Control negativo (Escherichia coli). M: marcador de peso molecular.

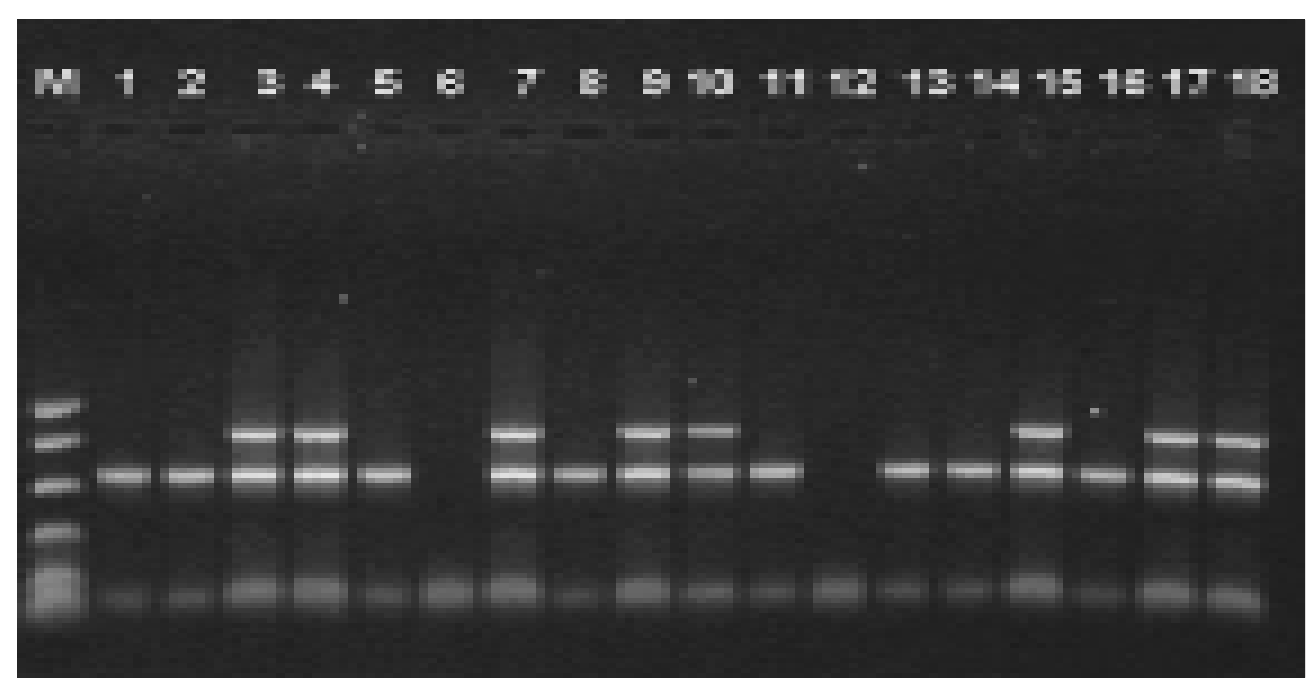

Figura 2. Diferenciación entre $M$. tuberculosis, M. bovis y M. bovis BCG. El fragmento de 1033 (Rv1510) pb está presente sólo en M. tuberculosis, permitiendo diferenciarlo de $M$. bovis y $M$. bovis BCG. La ausencia del fragmento de $999 \mathrm{pb}$ (Rv3877/78) en M. bovis BCG, permite diferenciar $M$. bovis BCG de M. bovis. Este fragmento también está presente en $M$. tuberculosis, pero la discriminación con este miembro del complejo la define el gen Rv1510. M: Marcador de peso molecular, se muestran sólo las dos primeras bandas. Mtb: M. tuberculosis, Mb: M. bovis (no BCG), MbBCG: M. bovis BCG.

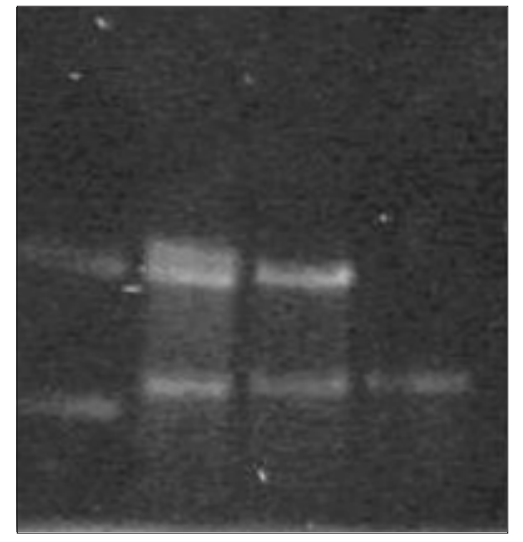

(Figura 2), permitiendo diferenciar M. bovis variedad BCG de M. bovis y de M. tuberculosis.

Es importante destacar que en este ensayo de RPC múltiple se incluyeron las secuencias de los genes Rv1510 (RD4) y Rv3877/8 (RD1) para diferenciar entre M. tuberculosis, $M$ bovis y M. bovis variedad BCG, pero también se incorporaron secuencias dirigidas al gen Rv0577, con la finalidad de disponer de un ensayo que permita en una única reacción de RPC, detectar y diferenciar estos miembros del complejo M. tuberculosis, obviando la reacción de RPC doble. En múltiples intentos de estandarizar la amplificación simultánea con los cuatro pares de oligonucleótidos (16S ARNr, Rv0577, Rv1510 y Rv3877/8), aparecieron múltiples bandas inespecíficas.

\section{Discusión}

bovis variedad BCG) de otros miembros del complejo $\mathrm{o}^{23}$, $\mathrm{y}$ en particular, en este estudio permitió diferenciar $M$. bovis y $M$. bovis variedad BCG de M. tuberculosis, que representan las especies de importancia clínica y epidemiológica en nuestro medio.

Para discriminar entre $M$. bovis y $M$. bovis variedad BCG en el ensayo de RPC múltiple, se incluyó otro par de oligonucleótidos partidores dirigido a los marcos de lectura Rv3877/Rv3878 (locus RD1) ${ }^{19,20,23}$, que amplificaron un producto de $999 \mathrm{pb}$ en M. bovis (también en M. tuberculosis), pero no en M. bovis variedad BCG
En los últimos años se han diseñado algunos ensayos de amplificación por RPC, basados en diferencias genotípicas entre especies micobacterianas ${ }^{26-31}$, y algunos se encuentran comercialmente disponibles ${ }^{12-14}$. Sin embargo, todos estos ensayos son estandarizados bajo condiciones específicas de los laboratorios donde fueron desarrollados, con protocolos de extracción y de amplificación de ácidos nucleicos, que muchas veces no son reproducibles bajo las condiciones propias de los laboratorios locales. En el caso de los kits comerciales, además de sus costos elevados, las se- 
cuencias de ADN utilizadas hasta el presente no permiten distinguir entre miembros individuales del complejo M. tuberculosis, lo cual limita su uso con propósitos de rutina de identificación.

Partiendo de la información de regiones de diferencia (RD) del genoma en algunas subespecies del complejo M. tuberculosis, de un panel de tipificación previamente publicado ${ }^{16-23}$, y de un formato de RPC doble utilizado en el laboratorio ${ }^{24}$, se estandarizó y aplicó un segundo ensayo de RPC múltiple que resultó de un gran valor en el laboratorio para detectar aislados clínicos del complejo $M$. tuberculosis y diferenciar entre M. tuberculosis, M. bovis y M. bovis variedad BCG.

Es importante resaltar que la secuencia del gen Rv1510 (RD4) fue propuesta originalmente para diferenciar $M$. bovis (incluyendo M. bovis variedad BCG) de otros miembros del complejo M. tuberculosis, debido a que $M$. bovis es el único miembro del complejo que carece del locus $\mathrm{RD}^{23}$. Igualmente, se propuso que la ausencia de los genes Rv3877 y Rv3878 (fragmento de $999 \mathrm{pb}$ ) puede ser utilizada como marcador para diferenciar $M$. bovis variedad BCG de otras subespecies, por ser el único miembro del complejo que carece del locus RD123. Sin embargo, el espectro diagnóstico propuesto en este trabajo se concentró en la identificación diferencial de $M$. tuberculosis, M. bovis y $M$. bovis variedad BCG, debido a que éstas son las micobacterias de importancia clínica y epidemiológica en nuestra región; de hecho, las otras micobacterias del complejo $M$. tuberculosis, tales como M. africanum (subtipos I y II), M. caprae, M. microti y M. canettii, no han sido aisladas en el laboratorio de referencia de diagnóstico. .

De acuerdo con lo anterior, el poder discriminatorio del ensayo propuesto, debe ser valorado integralmente al evaluar, en su conjunto, el patrón de amplificación obtenido con los tres pares de oligonucleótidos incluidos, lo cual podría expresarse, en una forma simplificada, de acuerdo a la presencia (+) o ausencia (-) de los genes Rv0577, Rv1510, Rv3877/78, respectivamente, de la siguiente manera: $M$. tuberculosis $(+++), M$. bovis $(+-+)$ y M. bovis variedad BCG (+ - -).

La aplicación de estos ensayos sobre aislados clínicos demostró que los oligonucleótidos utilizados superan el valor diagnóstico de otras secuencias ampliamente utilizadas. Por ejemplo, algunos intentos para diferenciar entre miembros del complejo M. tuberculosis incluye el diseño de ensayos de RPC dirigidos al elemento de inserción IS6110 ${ }^{26}$, basados en la observación de que $M$. tuberculosis contiene más copias de IS6110 que $M$. bovis ${ }^{27}$, pero estudios posteriores demostraron que algunas cepas de $M$. bovis y $M$. tuberculosis, exhibían elevado y bajo número de copias de IS6110, respectivamente ${ }^{28-30}$. Igualmente, se ha demostrado que la secuencia del gen $m t p 40$, también propuesta y utilizada en ensayos de identificación $^{31}$, está ausente en algunos aislados clínicos de $M$. tuberculosis ${ }^{32}$.

Este protocolo de identificación puede ser orientado al tomar en cuenta datos epidemiológicos; por ejemplo, en nuestro medio, $M$. bovis fue aislado predominantemente de personas que viven o laboran en regiones de intensa actividad agropecuaria, mientras que M. bovis variedad BCG fue aislada de pacientes inmunocomprometidos. Este ensayo cobra gran relevancia por el incremento en el número de pacientes con VIH co-infectados con $M$. bovis o $M$. bovis variedad BCG, detectados en nuestra región. Se debe destacar que la infección por $M$. bovis tiene gran importancia desde el punto de vista terapéutico, debido a que su patrón de susceptibilidad a medicamentos antituberculosos difiere al de $M$. tuberculosis; por ejemplo, la mayoría de las cepas de $M$. bovis tienen resistencia natural a pirazinamida, una de los fármacos de primera línea utilizadas en el tratamiento de la tubercu$\operatorname{losis}^{6-8}$, de manera que la identificación de aislados clínicos del complejo M. tuberculosis, particularmente la diferenciación entre especies $M$. tuberculosis y $M$. bovis, es un aspecto clave para un tratamiento adecuado del paciente y para brindar información epidemiológica a las Coordinaciones de los Programas de Control de Tuberculosis.

Aunque en este trabajo se seleccionaron secuencias genómicas que forman parte de un panel de tipificación desarrollado previamente, la especificidad de cada par de oligonucleótidos había sido evaluada sólo de manera individual ${ }^{23}$, mientras que en este trabajo se estandarizaron ensayos de amplificación simultánea, incorporando tres pares de oligonucleótidos y se aplicó el ensayo de RPC múltiple para la identificación diferencial de $M$. tuberculosis, M. bovis y $M$. bovis variedad BCG. Aunque los fragmentos de 1.034 pb y 999 pb difieren apenas en 34 pares de bases, éstos son perfectamente distinguibles en geles de agarosa al incrementar el tiempo de corrida electroforética, lo cual confiere gran versatilidad y sencillez de ejecución al ensayo.

Lamentablemente, no fue posible estandarizar condiciones de amplificación para incorporar los cuatro pares de oligonucleótidos seleccionados en este estudio en el formato de RPC múltiple, debido a que en varios intentos, cambiando concentración de oligonucleótidos y de $\mathrm{MgCl}_{2}$, o ensayando varias temperaturas de alineamiento, se observó la aparición de múltiples bandas inespecíficas y/o fallas en amplificar secuencias del gen Rv0577.

El protocolo de RPC fue validado en el laboratorio de biología molecular, al encontrar una correlación de 
$100 \%$ con la identidad de los aislados clínicos del complejo $M$. tuberculosis -establecida por cultivo y caracterización bioquímica en el laboratorio de referencia de diagnóstico de tuberculosis-, debido a que fueron obtenidos patrones de amplificación esperados para cada miembro del complejo. Dado el poder discriminatorio de estos oligonucleótidos, este protocolo de RPC podría facilitar el trabajo de laboratorios de referencia para el diagnóstico de tuberculosis, en la identificación rápida de micobacterias o complementar los ensayos de caracterización bioquímica a nivel de especie. De esta manera, contribuirían a acortar el período de diagnóstico, lo cual tiene un gran impacto en la elección de un tratamiento adecuado y oportuno para el paciente. Adicionalmente, el ensayo podría ser aplicado en programas de control de calidad de productos animales con sospechas de infección y estudios a gran escala de transmisión zoonótica de $M$. bovis desde animales a humanos.

\section{Agradecimientos}

Al Consejo de Desarrollo Científico y Humanístico de la Universidad del Zulia por el co-financiamiento de esta investigación. A la Oficina de Planificación del Sector Universitario (OPSU) por el fortalecimiento de la infraestructura del laboratorio de Biología Molecular.

\section{Resumen}

Las micobacterias que causan tuberculosis en animales y humanos pertenecen al complejo Mycobacterium tuberculosis. Las técnicas de diagnóstico convencional, además de ser lentas y laboriosas, no permiten diferenciar entre miembros de este complejo. El objetivo de este estudio fue evaluar ensayos de RPC múltiple para contribuir a la identificación diferencial de micobacterias del complejo M. tuberculosis a partir de cultivos, en un laboratorio de referencia. Se utilizaron oligonucleótidos partidores basados en regiones de diferencia (RD) que consisten en segmentos de ADN que están presentes en $M$. tuberculosis, pero que han sido eliminados diferencialmente del genoma de otros miembros del complejo M. tuberculosis. El ensayo se aplicó sobre 86 aislados clínicos de micobacterias. El patrón de amplificación permitió diferenciar entre cepas de $M$. tuberculosis, M. bovis y $M$. bovis variedad BCG en una única RPC. Este ensayo de RPC múltiple puede ser utilizado en el Laboratorio de Referencia de Diagnóstico de Tuberculosis como prueba complementaria para diferenciar micobacterias del complejo $M$. tuberculosis, contribuyendo a un acortamiento en el período de reporte de resultados y un tratamiento adecuado del paciente, y podría ser aplicado también en estudios epidemiológicos de transmisión zoonótica de $M$. bovis a humanos.

\section{Referencias}

1.- Van Soolingen D, Hoogenboezem T, de Haas P E, Hermans P W, Koedam M A, Teppema K S, et al. A novel pathogenic taxon of the Mycobacterium tuberculosis complex, Canetti: characterization of an exceptional isolated from Africa. Int $\mathrm{J}$ Syst Bacteriol 1997; 47: 1236-45.

2.- Gutierrez M, Samper S, Jimenez M S, van Embden J D, Marín J F, Martín C. Identification for spoligotyping of a caprine genotype in Mycobacterium bovis strain causing human tuberculosis. J Clin Microbiol 1997; 35: 3328-30.

3.- Horstkotte M A, Sobottka I, Schewe C K, Scafer P, Laufs R, Rusch-Gerdes S, et al. Mycobacterium microti llama-type infection presenting as pulmonary tuberculosis in a human immunodeficiency virus positivepatient. J Clin Microbiol 2001; 39: 406-7.

4.- Organización Panamericana de la Salud. Oficina Regional de la OMS. Situación epidemiológica de la tuberculosis en las Américas. Hoja Informativa 2004, Américas 2004; p 13-9.
5.- Ministerio de Salud y Desarrollo Social. Anuario de Mortalidad 2003. República Bolivariana de Venezuela. 2004 p 9-13.

6.- Ayele W Y, Neill S D, Zinsstag J, Weiss M G, Pavlik I. Bovine tuberculosis: an old disease but a new threat to Africa. Int J Tuberc Lung Dis 2004; 8: 924-37.

7.- Fritsche A, Engel R, Buhl D, Zellweger J P. Mycobacterium bovis tuberculosis: from animal to man and back. Int J Tuberc Lung Dis 2004; 8: 903-4.

8.- Zumárraga M J, Martin C, Samper S, Alito A, Latini O, Bigi F, et al. Usefulness of spoligotyping in molecular epidemiology of Mycobacterium bovis-related infections in South America. Microbiology 1999; 145: 893-7.

9.- Hesseling A C, Schaaf H S, Victor T, Beyers N, Marais B J, Cotton M F, et al. Resistant Mycobacterium bovis Bacillus Calmette-Guerin disease: implications for management of Bacillus Calmette-Guerin disease in human immunodeficiency virusinfected children. Pediatr Infect Dis J 2004; 5: 476-9.

10.- Samper S, Martín C, Pinedo A., Rivero A,
Blásquez J, Baquero F, et al. Transmission between HIV-infected patients of multidrugresistant tuberculosis caused by Mycobacterium bovis. AIDS 1997; 11: 1237-42.

11.- Koneman E, Allen D, Janda M, Scheckenberger P, Winn W. Mycobacteria. En E Koneman, D Allen, M. Janda, P Scheckenberger Eds. Colour Atlas and Textbook of Diagnostic Microbiology. Philadelphia: JB Lippincott Co; 2000, p 703-55.

12.- Piersimoni C, Scarparo C. Relevance of commercial amplification methods for direct detection of Mycobacterium tuberculosis complex in clinical samples. J Clin Microbiol 2003; 41: 5355-65.

13.- Eing B R, Becker A, Sohns, A, Ringelmann R. Comparison of Roche Cobas Amplicor Mycobacterium tuberculosis assay with in-house PCR and culture for detection of M. tuberculosis. J Clin Microbiol 1998; 36: 2023-9.

14.- Katila L, Schouls L, ErkinjunttiPerkkanen R. Accelerated detection and identification of mycobacteria with MGIT 
960 and COBAS Amplicor systems. J Clin Microbiol 2000; 38: 960-4.

15.- Brosch R, Gordon S V, Marmiesse M, Brodin P, Buchrieser C, Eiglmeier K, et al. A new evolutionary scenario for the Mycobacterium tuberculosis complex. Proc Natl Acad Sci USA, 2002; 99: 3684-9.

16.- Mostowy S, Cousins D, Brinkman J, Aranaz A, Behr M A. Genomics deletions suggest a phylogeny for the Mycobacterium tuberculosis complex. J Infect Dis 2002; 186: 74-80.

17. - Liebana E, Aranaz A, Francis B, Cousins D. Assessment of genetic markers for species differentiation within the Mycobacterium tuberculosis complex. J Clin Microbiol 1996; 34: 933-8.

18.- van Embden J D, van Gorkom D T, Kremer K, Jansen R, van der Zeijst B A, Shouls L M. Genetic variation and evolutionary origin of the direct repeat locus of Mycobacterium tuberculosis complex bacteria. J Bacteriol 2000; 182: 2393-401.

19.- Gordon S V, Brosch R, Billaut A, Garnier T, Eiglmeir K, Cole S T. Identification of variable regions in the genomes of tubercle bacilli using bacterial artificial chromosomes arrays. Mol Microbiol 1999; 32: 643-55.

20.- Mostowy S, Cousins D, Behr M A. Genomic interrogation of the dassie bacillus reveals it as a unique RD1 mutant within the Mycobacterium tuberculosis complex. J Bacteriol 2004; 186: 104-9.

21.- Park H, Jang H, Kim C, Chung B, Chan C,
Park SK, et al. Detection and identification of Mycobacteria by amplification of the internal transcribed spacer regions with genus-and species-specific PCR primers. J Clin Microbiol 2000; 38: 4080-5.

22.- Parsons L M, Brosch R, Cole S T, Somoskovi A, Loder A, Bretzel G, et al. Rapid and simple approach for identification of Mycobacterium tuberculosis complex isolates by PCR-based genomic deletions analysis. J Clin Microbiol 2002; 40: 223945.

23.- Huard R C, de Oliveira L C, Butler R, van Soolingen D, Ho J L. PCR-based method to differentiate the subspecies of the Mycobacterium tuberculosis complex on the basis of genomic deletions. J Clin Microbiol 2003; 41: 1637-50.

24.- Arráiz N, Bermúdez V, Romay Z, Faría N. Mujica D. Evaluation of a duplex PCR assay for the identification of Mycobacterium tuberculosis complex and nontuberculous Mycobacteria. Rev Cient FCV-LUZ 2005; XV (6): 568-75.

25.- Ausubel F M. Chapter 2. Preparation and Analysis of DNA. Short Protocols in Molecular Biology. 2nd ed. New York, USA, John Wiley \& Sons, Inc; 1992; p 2.10-32.

26.- Suffys P, Palomino JC, Cardoso S, Espitia C, Cataldi A, Alito A. Evaluation of the polymerase chain reaction for the detection of Mycobacterium tuberculosis. Int J Lung Dis 2000; 4: 179-83.

27.- Plikaytis B B, Eisenach K D, Crowford J T, Shinnick T M. Differentiation of
Mycobacterium tuberculosis and Mycobacterium bovis BCG by a polymerase chain reaction assay. Mol Cell Probes 1991; 5: 215-9.

28.- Torrea G, van De Perre P, Ouedraogo M, Zougba A, Sawadogo A, Dingtoumda B, et al. PCR-based detection of the Mycobacterium tuberculosis complex in urine of HIVinfected and uninfected pulmonary and extrapulmonary tuberculosis patients in Burkina Faso. J Med Microbiol 2005; 54: 39-44.

29.- Kent L, McHugh T D, Billington O, Dale J W, Gillespie SH. Demonstration of homology between IS6110 of Mycobacterium tuberculosis and DNAs of other Mycobacterium spp. J Clin Microbiol 1995; 33: 2290-93.

30.- El-Dawi T G, Saeedel N S, Hamid M E. Evaluation of a PCR-amplified IS6110 insertion element in the rapid diagnosis of pulmonary tuberculosis in comparison to microscopic methods in Sudan. Saudi Med J 2004; 25: 1644-7.

31.- Parra C A, Londoño I P, Del Portillo P, Patarroyo M E. Isolation, characterization, and molecular cloning of a specific mycobacterium tuberculosis antigen gene: identification of a species-specific sequence. Infect Immun 1991; 59: 3411-7.

32.- Weil A, Plikaytis B B, Butler W R, Wodley C I, Shinnick T M. The mtp40 gene is not present in all strain of Mycobacterium tuberculosis. J Clin Microbiol 1996; 34: 2309-11. 